\title{
Editorial \\ Suppression of bone morphogenetic protein inhibitors promotes osteogenic differentiation: therapeutic implications
}

\author{
Manolis Heliotis ${ }^{1}$ and Eleftherios Tsiridis ${ }^{2}$
}

\begin{abstract}
${ }^{1}$ Regional North West London Maxillofacial Unit, Northwick Park Hospital, Wattford Road, Harrow, London HA1 3UJ, UK
${ }^{2}$ Academic Orthopaedic Unit, Institute of Molecular Medicine, Leeds Medical School, and Leeds General Infirmary Teaching Hospital, Clarendon, Great George Street, Leeds LS1 3EX, UK
\end{abstract}

Corresponding author: Eleftherios Tsiridis, etsiridis@doctors.org.uk

Published: 12 August 2008

This article is online at http://arthritis-research.com/content/10/4/115

(c) 2008 BioMed Central Ltd

See related research article by Kwong et al., http://arthritis-research.com/content/10/3/R65
Arthritis Research \& Therapy 2008, 10:115 (doi:10.1186/ar2467)

\begin{abstract}
Bone morphogenetic proteins (BMPs) are expressed during osteogenesis and their action is regulated by corresponding BMP inhibitors. Chordin (a well recognized BMP inhibitor) and BMP-2 are expressed during osteogenic differentiation of human mesenchymal stem cells. Chordin inhibition induces human mesenchymal stem cell differentiation and reduces their proliferation by increasing BMP-2 bioavailability. The potential of suppressing BMP inhibitors is emerging as a biological therapeutic target in bone tissue engineering, because it results in an unopposed synergy between the various growth factors that are involved in osteogenesis, within their physiological milieu.
\end{abstract}

Bone morphogenetic proteins (BMPs), a distinct transforming growth factor (TGF)- $\beta$ subgroup that is also involved in organogenesis, have been shown to be expressed during various stages of bone healing process. Mesenchymal stem cells (MSCs) that are capable of differentiation toward the osteogenic lineage are recruited from local deposits or the circulation to initiate callous formation. Kwong and coworkers [1], in their recent report, presented the expression of BMP-2 and its inhibitor chordin in human MSCs undergoing differentiation in an osteogenic medium. Temporal and spatial expression of BMPs, has attracted research interest because a number of its members, namely BMP-2, $-7,-9$ and -14 , can induce de novo bone formation [2].

BMPs bind to two different type I and type II serine/threonine kinase receptors. BMP heteromeric receptor complexes activate intracellular proteins known as Smads (small 'mothers against' decapentaplegic), including regulating $\mathrm{R}$-Smads (Smad1, Smad5 and Smad8), common co-Smad (Smad4), and inhibitory I-Smads (Smad6 and Smad7), which translocate to the nucleus activating specific target genes [3].

BMP inhibitors regulate local negative feedback mechanisms. Some act extracellularly, preventing BMP binding to receptors, whereas others act intracellularly, inhibiting Smad downstream activation.

The most studied BMP inhibitor, noggin, reduces the activity of both osteoblasts and osteoclasts, leading to osteopenia and nonhealing fractures. In contrast, noggin knockout leads to irregularly thickened long bones and is lethal shortly after birth [4]. Chordin has a similar action to noggin. It antagonizes BMP-2, -4 and -7 by preventing them from activating BMP receptors, augmented by the co-factor twisted gastrulation (Tsg) [5]. This causes a decrease in osteogenic differentiation and a reduction in BMP-2 and BMP-4 expression $[1,5]$. Gremlin, another extracellular inhibitor of BMP-2, -4 and -7 engineered in transgenic mice, suppresses osteoblasts with consequent osteopenia and spontaneous fractures [6]. Sclerostin, a transcription factor inhibitor, promotes apoptosis of human MSCs and osteoblasts. Interestingly, sclerostin and noggin - when they are expressed together - form a mutually inhibitory complex that actually leads to promotion of BMP activity [7]. Inhibitory Smad6 and Smad7 are found predominantly in the cell nucleus, but they are transported into the cell cytoplasm at times of high BMP activity. Smad6 preferentially inhibits BMP signaling, whereas Smad7 inhibits all members of the TGF- $\beta$ family [3]. Overall, BMP inhibitors lead to downregulation of BMPs, inhibition of MSC differentiation to osteoblasts, and poor fracture healing. On the other

$\mathrm{BMP}=$ bone morphogenetic protein; MSC $=$ mesenchymal stem cell; Smad = small 'mothers against' decapentaplegic; TGF $=$ transforming growth factor. 
hand, it has been demonstrated that the lack of BMP inhibitors in knockdown mice has an adverse effect on development of the musculoskeletal system [1,4-7].

The report by Kwong and coworkers [1] elegantly illustrated that a balance exists between chordin and BMP-2 expression during human MSC differentiation into osteoblasts [1]. They found that both chordin and BMP-2 increase during osteogenic differentiation of MSCs, but their effects are markedly different. The suppression of chordin led to increased availability of BMP-2 and subsequently differentiation of human MSCs into osteoblasts. Those investigators further demonstrated that suppression of chordin not only promoted osteogenesis (differentiation) but also decreased cellular proliferation, also mediated by BMP-2. Therefore, an inverse relation exists between these two processes.

By inhibiting other inhibitors, some interesting recent studies have also demonstrated in vitro and in vivo osteogenesis. Wan and colleagues [8] identified that accelerated bone formation resulted from suppressing noggin expression in both in vitro and in vivo experiments. After transfected osteoblasts (small-interfering RNA noggin) had been implanted into critically sized parietal bone defects of skeletally mature mice early ( 2 weeks), bone regeneration took place, with nearly complete bony bridging by 8 weeks. Winkler and coworkers used mouse C3H10T1/2 cells treated with rh-noggin and transfected with bacillovirus-produced sclerostin. They showed that combining noggin and sclerostin at a specific concentration resulted in a single complex, neutralizing their respective inhibitory effects. This allowed BMP signalling to proceed to osteoinduction [7].

The multitude and complexity of BMP, TGF- $\beta$ and vascular endothelial growth factor synergy in bone regeneration is such that the application of a single or at most two commercially manufactured growth factors cannot be the solution to the problem of inadequate bone healing. In addition, high concentrations - at high cost - of single growth factors with a range of questionable carriers is the current paradigm for bone induction in the clinical setting [9]. The potential of inhibiting the inhibitors is therefore of great interest, because a single inhibitor - such as noggin or chordin - controls several BMPs (specifically BMP-2, -4 and -7) and therefore allows their natural synergy to regenerate bone in a physiological state. This takes advantage of the endogenous BMP cascade both temporally and spatially $[1,4-8]$. Also, suppression of BMP inhibitors could be a potential therapy for osteoporosis [10].

A strength of the report by Kwong and coworkers [1] is its recognition of the role played by chordin in osteogenesis. The authors demonstrated successful knockdown of chordin expression by small-interfering RNA transfection of human MSCs cultured in an osteogenic medium, and they concluded that chordin suppression allows MSC differentiation by increasing BMP-2 bioavailability. Future characterization of all the inhibitors and technological advances in suppressing their expression (including gene therapy, small interfering RNA induced knockdown inhibitor expression, neutralizing antibodies and small molecule antagonists) will allow noninvasive 'intelligent' bone tissue engineering.

\section{Competing interests}

The authors declare that they have no competing interests.

\section{References}

1. Kwong FNK, Richardson SM, Evans $\mathrm{CH}$ : Chordin knockdown enhances the osteogenic differentiation of human mesenchymal stem cells. Arthritis Res Ther 2008, 10:R65.

2. Cho TJ, Gerstenfeld LC, Einhorn TA: Differential temporal expression of members of the transforming growth factor beta superfamily during murine fracture healing. $J$ Bone Miner Res 2002, 17:513-520.

3. Sakou T, Onishi T, Yamamoto T, Nagamine T, Sampath T, Ten Dijke P: Localization of Smads, the TGF-beta family intracellular signaling components during endochondral ossification. $J$ Bone Miner Res 1999, 14:1145-1152.

4. Groppe J, Greenwald J, Wiater E, Rodriguez-Leon J, Economides AN, Kwiatkowski W, Affolter M, Vale WW, Belmonte JC, Choe S: Structural basis of BMP signalling inhibition by the cystine knot protein Noggin. Nature 2002, 12:636-642.

5. Chang C, Holtzman DA, Chau S, Chickering T, Woolf EA, Holmgren LM, Bodorova J, Gearing DP, Holmes WE, Brivanlou AH: Twisted gastrulation can function as a BMP antagonist. Nature 2001, 22:483-487

6. Gazzerro E, Pereira RC, Jorgetti V, Olson S, Economides AN, Canalis E: Skeletal overexpression of gremlin impairs bone formation and causes osteopenia. Endocrinology 2005, 146: 655-665.

7. Winkler DG, Yu C, Geoghegan JC, Ojala EW, Skonier JE, Shpektor D, Sutherland MK, Latham JA: Noggin and sclerostin bone morphogenetic protein antagonists form a mutually inhibitory complex. J Biol Chem 2004, 279:36293-36298.

8. Wan DC, Pomerantz JH, Brunet LJ, Kim JB, Chou YF, Wu BM, Harland R, Blau HM, Longaker MT: Noggin suppression enhances in vitro osteogenesis and accelerates in vivo bone formation. J Biol Chem 2007, 282:26450-26459.

9. Tsiridis E, Ali Z, Bhalla A, Heliotis M, Gurav N, Deb S, DiSilvio L: In vitro and in vivo optimization of impaction allografting by demineralization and addition of rh-OP-1. J Orthop Res 2007, 25:1425-1437.

10. Wu XB, Li Y, Schneider A, Yu W, Rajendren G, lqbal J, Yamamoto M, Alam M, Brunet LJ, Blair HC, Zaidi M, Abe E: Impaired osteoblastic differentiation, reduced bone formation, and severe osteoporosis in noggin-overexpressing mice. J Clin Invest 2003, 112:924-934. 